\section{Effect of Shading on Anthocyanin and Non-flavonoid Polyphenol Biosynthesis of Gynura bicolor Leaves in Midsummer}

\author{
Nobuyuki Fukuoka ${ }^{1}$ \\ Attached Farm, Ishikawa Prefectural University, 1-308, Suematsu, Nonoichi, \\ Ishikawa 921-8836, Japan
}

Takamoto Suzuki

Department of Food Science, Ishikawa Prefectural University, 1-308, Suematsu, Nonoichi, Ishikawa 921-8836, Japan

\section{Keisuke Minamide}

Research Institute for Bioresources and Biotechnology, Ishikawa Prefectural University, 1-308, Suematsu, Nonoichi, Ishikawa 921-8836, Japan; and Ishikawa Agricultural Research Center, 295-1, Saitamachibo, Kanazawa, Ishikawa 9203198, Japan

\section{Tatsuro Hamada}

Research Institute for Bioresources and Biotechnology, Ishikawa Prefectural University, 1-308, Suematsu, Nonoichi, Ishikawa 921-8836, Japan

Additional index words. anthocyanin, chlorogenic acids, Gynura bicolor, shading

\begin{abstract}
The effect of shading in midsummer on anthocyanin and non-flavonoid polyphenol biosynthesis of Gynura bicolor DC leaves was examined using a control (full solar radiation) and a shade treatment $(50 \%$ shading of full solar radiation). Leaf temperature in the shade plot remained $\approx 40{ }^{\circ} \mathrm{C}$ in the daytime, $\approx 6{ }^{\circ} \mathrm{C}$ lower than in the control. Plants in the shade plot grew better than the control. The content of chlorogenic acid (CGA) in leaves decreased with leaf maturation for both treatments, and a larger amount of CGA was detected in leaves from the control than the shade treatment. The profiles of reactive oxygen species (ROS) scavenging activity exhibited an identical pattern to the content of CGA. Although there was an abrupt increase in the content of anthocyanin in the early stage of leaf expansion, the content decreased rapidly as the leaves matured. The increase in anthocyanin early during leaf expansion was much more limited in control leaves than shaded leaves. There were no correlation between the profiles of anthocyanin and gene expression such as $G b P A L, G b C 4 H, G b 4 C L, G b C H S$, $G b C H I, G b F 3 H$, and $G b U F G T$. However, the profiles of expression of genes such as $G b M Y B 2, G b F 3^{\prime} H, G b D F R$, and $G b A N S$ were similar to the anthocyanin profiles. These results suggest that artificial shading in midsummer is an effective method to promote anthocyanin accumulation but reduces ROS scavenging capacity as a result of lowered CGA production.
\end{abstract}

Gynura bicolor DC is widely distributed in certain areas of southeast Asia. It is a member of the Asteraceae family and has several local names, e.g., Suizenjina and Kinjisou in Japan (Hayashi et al., 2002). Its leaves are characterized by green adaxial and reddish purple abaxial surfaces. In Ishikawa Prefecture, G. bicolor is cultivated in the field from early spring to late fall. However, the reddish purple abaxial sides of leaves harvested in midsummer often lack this characteristic color (Yoshiaki et al., 2010).

Received for publication 1 May 2014. Accepted for publication 13 July 2014.

${ }^{1}$ To whom reprint requests should be addressed; e-mailnfukuoka@ishikawa-pu.ac.jp. grape cultivar (Mori et al., 2007). Because G. bicolor plants are often exposed to high temperature in midsummer, the loss of red color of the leaves may largely be dependent on a repression of anthocyanin biosynthesis genes caused by high temperature.

Chlorogenic acid is the most abundant phenolic compound in $G$. bicolor leaves (Fukuoka et al., 2012). The contents increase in response to environmental stresses (Oh et al., 2009). In lettuce, leaves of sun-exposed plants contained more CGA than leaves from plants growing under shaded locations (Grace et al., 1998). Generally, phenolic compounds including anthocyanin can scavenge ROS (Kanatt et al., 2005; Nara et al., 2006). Chlorogenic acid was the most efficient scavenger of free radicals in G. bicolor leaves (Fukuoka et al., 2012). Because stress conditions enhance the production of ROS (Asada and Takahashi, 1987), leaves from sun-exposed locations in midsummer may contain a larger amount of CGA to scavenge abundant ROS.

However, because G. bicolor is a minor crop, there has been little research on this plant and no reports of changes in polyphenol biosynthesis of leaves induced by shading. In this article, we show that artificial shading in midsummer is an effective method to promote anthocyanin accumulation but reduces ROS scavenging capacity as a result of lower production of CGA.

\section{Materials and Methods}

Plant materials and experimental plots. Cuttings ( $\approx 6$ to $9 \mathrm{~cm}$ long with three nodes) of Gynura bicolor shoots were obtained from mother plants on 4 June and planted in germination boxes filled with sandy soil and then grown in a greenhouse. Approximately $20 \mathrm{~d}$ after cutting, plants were transplanted into $10.5-\mathrm{cm}$ polypots filled with clay soil containing various mixtures of organic manure. Primary shoots were pinched off $20 \mathrm{~d}$ after transplanting, and then the plants were grown under natural conditions until two foliage leaves had fully expanded. Lateral shoots that emerged were reduced to three on each cutting $10 \mathrm{~d}$ after pinching, and the seedlings were planted in the field at $30-\mathrm{cm}$ intervals in rows $110 \mathrm{~cm}$ apart. A basal fertilizer $(10 \mathrm{~N}-10 \mathrm{P}-10 \mathrm{~L})$ of $100 \mathrm{~g} \cdot \mathrm{m}^{-2}$ was applied before planting. Microelemental manure $\left(60 \mathrm{~g} \cdot \mathrm{m}^{-2}\right)$ containing $0.4 \%$ manganese, $0.3 \%$ boron, $1.2 \%$ iron, and $0.03 \%$ copper was added at the same time.

To clarify the effect of shading on polyphenol biosynthesis, plants were grown in two experimental plots, a control and a shade treatment. Control plants were grown under the usual conditions in midsummer. For the shade treatment, tunnels $(90 \mathrm{~cm}$ high $\times 120 \mathrm{~cm}$ wide) were placed above the plants ( 100 plants per plot) and covered with an SL50 silver nylon mesh (Diatex Co., Ltd., Tokyo, Japan) that provided $50 \%$ shading of full solar radiation. Changes in daily light intensity and leaf temperature at the top of the canopy were respectively measured on a clear day (2 Aug.) using a TM-201 illuminometer (Tenmars 
Electronics Co., Ltd., Taipei, Taiwan) and a TA-0510bF radiation thermometer (Minolta Camera Co., Ltd., Osaka, Japan). On 16 Aug., $\approx 50$ lateral shoots were harvested and 10 uniform shoots were used to measure plant growth. Subsequently, leaves were collected at one node intervals from the shoot apex to the basal end. Each collected leaf was numbered basipetally from the shoot apex as shown in Figure 1. A 3-g sample from each collected fresh leaf was cut into pieces and boiled in $40 \mathrm{~mL}$ distilled water for $10 \mathrm{~min}$. The mixture was filtered through cotton gauze and the extract was diluted to $50 \mathrm{~mL}$ with distilled water. The extracts were stored at $-45^{\circ} \mathrm{C}$ until analysis of phenolic compounds and antioxidant activity. The remaining fresh leaves were stored at $-80^{\circ} \mathrm{C}$ until extraction of RNA for analysis of anthocyanin biosynthetic gene expression.

Anthocyanin and CGA contents. Total phenol content was measured using the method described by Singleton et al. (1999) with some modifications. Briefly, a 3-fold volume of ethanol was added to $1.5 \mathrm{~mL}$ of the stored extract, and the precipitant was removed by centrifugation $(10,000 \mathrm{~g}$ for $15 \mathrm{~min})$. The ethanolic extract was dried at $60{ }^{\circ} \mathrm{C}$ and dissolved in $200 \mu \mathrm{L}$ of distilled water, and a $5-\mu \mathrm{L}$ aliquot of solution was brought up to $45 \mu \mathrm{L}$ with distilled water in each well of a 96-well microplate. Then, $50 \mu \mathrm{L}$ of Folin-Ciocalteau reagent and $50 \mu \mathrm{L}$ of $100 \mathrm{mg} \cdot \mathrm{L}^{-1} \mathrm{Na}_{2} \mathrm{CO}_{3}$ were added to each well. After incubation for $60 \mathrm{~min}$ at room temperature, the absorbance at $750 \mathrm{~nm}$ was measured using a plate reader (Bio-Rad Laboratories, Tokyo, Japan). Results were expressed as milligrams of gallic acid equivalent per gram fresh weight. All measurements were taken in triplicate and the mean values were calculated.

CGA is the most abundant phenolic compound in G. bicolor leaves (Fukuoka et al., 2012). Then, in this study, CGA was measured using the solution remaining after determination of total polyphenol content. A $10-\mu \mathrm{L}$ aliquot of this solution was injected into a Hitachi high-performance liquid chromatography (HPLC) system (Hitachi High-Tech Co. Ltd., Tokyo, Japan) equipped with an L-4200H ultraviolet detector (Hitachi High-Tech Co. Ltd.) and an Inertsil ODS-3 column $(150 \mathrm{~mm} \times 4.6 \mathrm{~mm} ; 5 \mu \mathrm{m}$ particle size; GL Sciences Inc., Tokyo, Japan). The mobile phase for HPLC consisted of a mixture of Solvent A ( $10 \mathrm{~mm} \mathrm{NaH}_{2} \mathrm{PO}_{4}$ in distilled water) and Solvent B (100\% methanol). The solvent gradient was as follows: Solvent B was increased from $10 \%(\mathrm{v} / \mathrm{v})$ to $50 \%(\mathrm{v} / \mathrm{v})$ in Solvent A over 20 min and then kept at $50 \%$ for the next $10 \mathrm{~min}$. The flow rate was 1.0 $\mathrm{mL} \cdot \mathrm{min}^{-1}$, the column temperature was $40^{\circ} \mathrm{C}$, and absorption was measured at $280 \mathrm{~nm}$. To identify the peak corresponding to CGA, CGA (Nacalai Tesque Inc., Kyoto, Japan) was used as external standard. Results were expressed as milligrams of CGA equivalent per gram fresh weight. All measurements were taken in triplicate and the mean values were calculated.

To determine the anthocyanin content, $\approx 3 \mathrm{~mL}$ of frozen leaf extract was extracted with a 3 -fold volume of $100 \%$ ethanol and the resulting precipitate was removed by centrifugation $(10,000 \mathrm{~g}$ for $15 \mathrm{~min})$. The ethanolic extract was then dried at $60{ }^{\circ} \mathrm{C}$ and dissolved in $3 \mathrm{~mL}$ of $0.1 \mathrm{M}$ citrate buffer ( $\mathrm{pH} 3.0$ ).
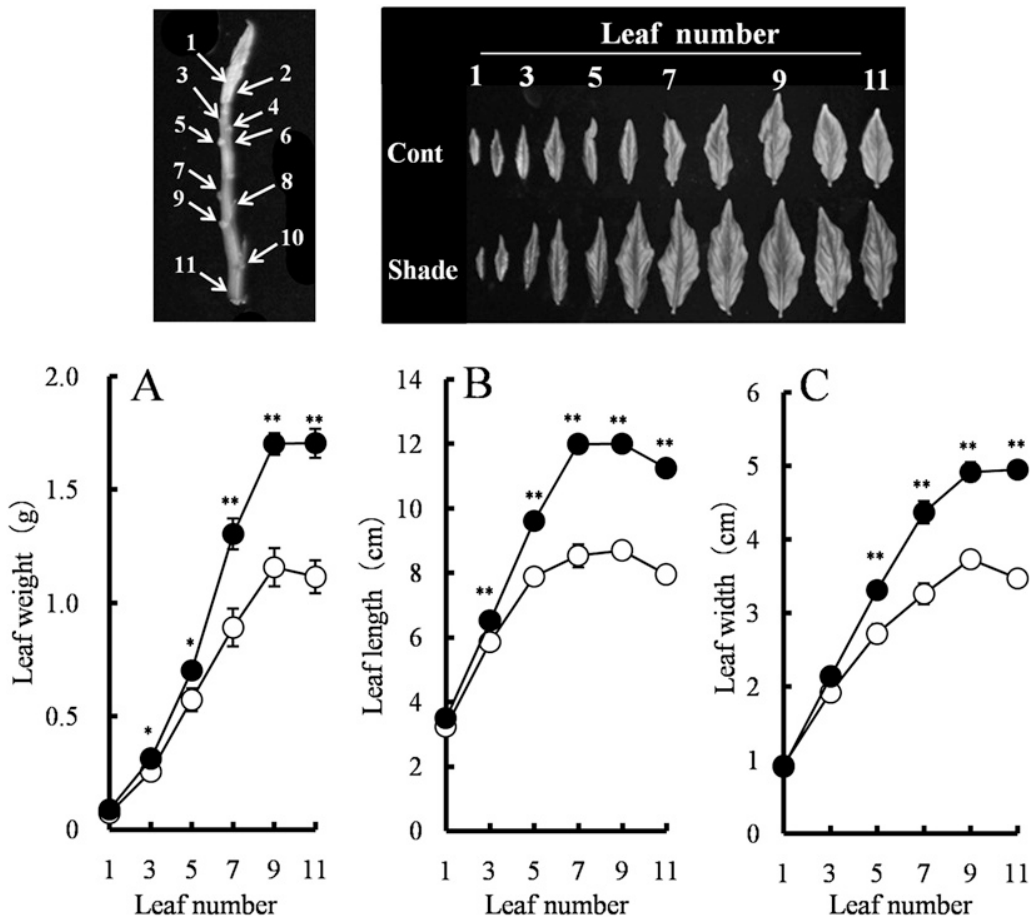

Fig. 1. Differences in leaf growth of G. bicolor plants between treatments. Unshaded and solid circles indicate control and shade treatments, respectively. (A) leaf weight; (B) leaf length; (C) leaf width. Vertical bars indicate \pm SE $(\mathrm{n}=10)$. **Significant differences at $P \leq 0.01$ by Student's $t$ test.

Anthocyanin content was measured as absorbance at $546 \mathrm{~nm}$ using a U-3900 ultravioletVis spectrophotometer (Hitachi Co., Ltd., Tokyo, Japan). Results were expressed as milligrams of equivalent cyanidin-3-glucoside per gram of fresh weight. All measurements were taken in triplicate and the mean values were calculated.

Analysis of anthocyanin biosynthetic gene expression. Approximately $800 \mathrm{mg}$ of frozen leaves for each sample were ground into powder in the presence of liquid nitrogen using a mortar and pestle. Approximately $300 \mathrm{mg}$ of each powder was transferred to sterile centrifuge tubes containing $4 \mathrm{~mL}$ Fruitmate for RNA purification reagent (Takara Bio Inc., Otsu, Shiga, Japan). The powder was thoroughly dispersed by hand shaking for a few seconds. After centrifugation at $9170 \mathrm{~g}$ for $5 \mathrm{~min}$ at $4{ }^{\circ} \mathrm{C}$, the supernatant was transferred to new polypropylene tubes and extracted with an equal volume of TRIzol reagent (Life Technologies, Inc., Gaithersburg, MD). After incubation for $5 \mathrm{~min}$ at room temperature, $0.2 \mathrm{~mL}$ of chloroform was added per milliliter of TRIzol reagent. The slurry was mixed vigorously and then centrifuged at $10,000 \mathrm{rpm}$ for $15 \mathrm{~min}$ at $4{ }^{\circ} \mathrm{C}$. Centrifugation separated the biphasic mixtures into a lower red phenol-chloroform phase and an upper colorless aqueous phase. RNA was precipitated from the aqueous phase by mixing with an equal volume of isopropanol. The liquid was incubated at room temperature for $10 \mathrm{~min}$ and centrifuged at $10,000 \mathrm{rpm}$ for $10 \mathrm{~min}$ at $4{ }^{\circ} \mathrm{C}$. The supernatant was removed and the RNA pellet was washed once with $75 \%(\mathrm{v} / \mathrm{v})$ ethanol. The pellet was air-dried and dissolved in $100 \mu \mathrm{L}$ Milli-Q water. The total RNA in the samples was quantified using an ND1000 spectrophotometer (Thermo Fisher Scientific, Inc., Wilmington, DE).

The RNA was purified on RNeasy mini columns (Qiagen, Chatsworth, CA), including on-column DNase I digestion as described by the manufacturer's protocol. RNA integrity was evaluated by viewing bands after agarose gel electrophoresis and staining with ethidium bromide. Aliquots of total RNA $(1 \mu \mathrm{g})$ were reverse-transcribed to cDNA using a ReverTra Ace qPCR RT Kit (Toyobo Co., Ltd., Osaka, Japan) as follows: denaturation at $65{ }^{\circ} \mathrm{C}$ for $5 \mathrm{~min}$, reverse transcription at $37^{\circ} \mathrm{C}$ for 15 $\min$ in a $10 \mu \mathrm{L}$ reaction containing $2 \mu \mathrm{L} 5 \times$ reverse transcription (RT) buffer, $0.5 \mu \mathrm{L}$ RT enzyme mix, $0.5 \mu \mathrm{L}$ primer mix, and the appropriate volume of nuclease-free water, and termination at $98^{\circ} \mathrm{C}$ for $5 \mathrm{~min}$. The cDNA samples obtained from these aliquots of total RNA were stored at $-20{ }^{\circ} \mathrm{C}$ until use.

The regulation of 10 genes of the anthocyanin biosynthetic pathway [phenylalanine ammonia-lyase $(G b P A L)$, cinnamic acid 4-hydroxylase $(\mathrm{GbC} 4 \mathrm{H})$, 4-coumarate $\mathrm{CoA}$ ligase $(G b 4 C L)$, chalcone synthase ( $G b C H S)$, chalcone isomerase $(G b C H I)$, flavonoid 3hydroxylase $\left(G b F 3^{\prime} H\right)$, flavanone 3-hydroxylase $(G b F 3 H)$, dihydroflavonol 4-reductase $(G b D F R)$, anthocyanidin synthase (GbANS)] and flavonoid 3-O-glucosyltransferase (GbUFGT) as well as two related transcription factors 
(GbMYB1, GbMYB2) was monitored, and GeneBank accession no. of them were AB550238, AB550251, AB550249, AB550239, AB550250, AB550253, AB550252, AB552040 AB550241, AB617731, AB552044, and AB552045, respectively. A 1:20 dilution of the resultant cDNA was used as the template to quantify the relative content of mRNA by real-time polymerase chain reaction (PCR) on a StepOnePlus system (Applied Biosystems, Foster City, CA) using the respective primers (Table 1). Each $19 \mu \mathrm{L}$ PCR mixture contained $1 \mu \mathrm{L}$ cDNA, $10 \mathrm{pmol} \cdot \mathrm{nL}^{-1}$ of each primer, and 10.2 $\mu \mathrm{L}$ GoTaq Green Master Mix (Promega Corp., Madison, WI). The PCR conditions were as follows: for the initial denaturation step, $95^{\circ} \mathrm{C}$ for $2 \mathrm{~min}$ followed by 40 cycles at $95{ }^{\circ} \mathrm{C}$ for $3 \mathrm{~s}, 60{ }^{\circ} \mathrm{C}$ for $30 \mathrm{~s}$, with a final elongation at $72{ }^{\circ} \mathrm{C}$ for $3 \mathrm{~min}$. The transcript levels of each gene were normalized to that of the tubulin gene (GeneBank accession no. AB581528) as a control. Relative gene expression was determined by the $\Delta \Delta C \mathrm{~T}$ method. All measurements were taken in triplicate and the mean values were calculated.

Antioxidant activities. Ferric-reducing power was measured using the eluant from the determination of total polyphenol content, which was diluted 5-fold with distilled water. Aliquots $(10 \mu \mathrm{L})$ of each solution were diluted to $25 \mu \mathrm{L}$ with distilled water in wells of a 96-well microplate. Approximately $25 \mu \mathrm{L}$ phosphate buffer $(0.1 \mathrm{M}, \mathrm{pH} 7.2)$ and $50 \mu \mathrm{L}$ $1 \%(\mathrm{w} / \mathrm{v})$ potassium ferricyanide were put into each well. After incubation at $37{ }^{\circ} \mathrm{C}$ for $60 \mathrm{~min}, 25 \mu \mathrm{L} 10 \%(\mathrm{w} / \mathrm{v}) \mathrm{TCA}, 100 \mu \mathrm{L}$ distilled water, and $25 \mu \mathrm{L} 0.1 \%(\mathrm{w} / \mathrm{v})$ ferric chloride $\left(\mathrm{FeCl}_{3}\right)$ were added to the mixture. An increase in absorbance at $620 \mathrm{~nm}$ of the reaction mixture indicated increased reducing power.

To determine hydroxyl radical scavenging activity, a 3-fold volume of ethanol was added to $1.5 \mathrm{~mL}$ of the stored extract and the polysaccharides were precipitated by centrifugation. The ethanolic extract was then dried at $60{ }^{\circ} \mathrm{C}$ and dissolved in $100 \mu \mathrm{L}$ distilled water. A $10-\mu \mathrm{L}$ aliquot of this solution was diluted to $100 \mu \mathrm{L}$ with distilled water in a well of a 96-well microplate, then $75 \mu \mathrm{L}$ phosphate buffer (0.025 M, pH 7.2), $25 \mu \mathrm{L}$ 1,10-phenanthroline $(0.006 \mathrm{M})$, and $25 \mu \mathrm{L}$ $\mathrm{FeSO}_{4}(0.006 \mathrm{M})$ were added. Hydroxyl radical was generated by adding $25 \mu \mathrm{L}$ $0.1 \%(\mathrm{v} / \mathrm{v}) \mathrm{H}_{2} \mathrm{O}_{2}$. The mixture was incubated at $37{ }^{\circ} \mathrm{C}$ for $60 \mathrm{~min}$ and the absorbance was measured at $540 \mathrm{~nm}$. Results were expressed as milligrams of catechin equivalent per gram fresh weight.

\section{Results}

Light intensity and leaf temperature. The daily maximum air temperature in the shade plot was $\approx 37^{\circ} \mathrm{C}, 2$ to $5^{\circ} \mathrm{C}$ lower than in the control (data not shown). Values of photosynthetic photon flux density $(P P F D)$ in the control increased rapidly after sunrise and reached $1760 \mu \mathrm{mol} \cdot \mathrm{m}^{-2} \cdot \mathrm{s}^{-1}$ at $1300 \mathrm{HR}$. On the other hand, the $P P F D$ values in the shade plot remained at $\approx 900 \mu \mathrm{mol} \cdot \mathrm{m}^{-2} \cdot \mathrm{s}^{-1}$ (data not shown). Results for leaf temperature were similar to those for $P P F D$. Leaf temperature in the shade plot increased rapidly after sunrise and reached $40{ }^{\circ} \mathrm{C}$ at $1300 \mathrm{HR}, \approx 6{ }^{\circ} \mathrm{C}$ lower than in the control (data not shown).

Plant growth. Plants in the shade plot grew better than the control. In the shade plot, the mean leaf weight for leaf number (LN) 5 was 0.7 and for LN 9, it was $1.7 \mathrm{~g}$, $\approx 122 \%$ and $147 \%$ of the respective values for the control (Fig. 1A). Leaf size showed a similar trend across treatments to that for leaf weight (Fig. 1B-C). The mean length and width of LN 9 in the shade plot were 12.0 and $4.9 \mathrm{~cm}$, respectively, $\approx 138 \%$ and $132 \%$ of the respective values in the control. Although the leaves in the shade plot had reddish purple abaxial surfaces, these surfaces in the control plant were lacking this characteristic color.

Anthocyanin and CGA contents. Total polyphenol content on a fresh weight basis decreased as leaves matured, and a lower content was detected in shaded leaves than control leaves, regardless of leaf stage (Fig. 2A). The polyphenol content of LN 3 and 7 in the shaded leaves was 2.1 and $1.6 \mathrm{mg} \cdot \mathrm{g}^{-1}$, respectively, which was $89 \%$ and $85 \%$ of the respective values in the control. The profile of fluctuation in CGA content exhibited an identical pattern with lower CGA content observed in the shaded leaves (Fig. 2B).
Although there was an abrupt increase in the content of anthocyanin in the early stage of leaf expansion, the content decreased rapidly as the leaves matured (Fig. 2C). The increase in anthocyanin early during leaf expansion was much more limited in control leaves than shaded leaves, although the decreased rate of anthocyanin accumulation in maturing leaves did not differ between treatments.

Anthocyanin biosynthetic genes. Twelve genes encoding GbMYB1, GbMYB2, GbPAL, GbC4H, Gb4CL, GbCHS, GbCHI, GbF3H, GbF3'H, GbDFR, GbANS, and GbUFGT were selected to examine the expression pattern of anthocyanin biosynthetic genes with respect to leaf age (Fig. 3). Expression of the transcription factor gene $G b M Y B 2$ was strongly intensified in the shaded leaves. Likewise, in structural genes such as $G b F 3^{\prime} H$, $G b D F R$, and GbANS, expression tended to decrease as the leaves matured, and the levels in LN 1 to 5 of the shaded leaves were much higher than those of comparable chronological age in the control. Conversely, the relationship between the profiles of fluctuation in expression of $G b 4 C L, G b C H S$, and $G b C H I$ and those of $G b F 3^{\prime} H, G b D F R$, and $G b A N S$ were mirror images of each other, and the levels of $G b 4 C L$, $G b C H S$, and $G b C H I$ transcripts showed no significant difference between the two experimental treatments, regardless of leaf stage.

Table 1. Primers for quantitative reverse transcription-polymerase chain reaction analysis.

\begin{tabular}{lll}
\hline Gene & \multicolumn{1}{c}{ Forward primer $\left(5^{\prime}\right.$ to $\left.3^{\prime}\right)$} & \multicolumn{1}{c}{ Reverse primer $\left(5^{\prime}\right.$ to $\left.3^{\prime}\right)$} \\
\hline$G b P A L$ & CTTACTTGACCGGCGAAAAGG & TTTGCACATAGCCGTGAACAC \\
$G b C 4 H$ & CGCTAAACTTGGCGGCTATG & GCTAACCACCACGCGTTGA \\
$G b 4 C L$ & GCCGAGATGAAGATCGTTGAC & CATATCTCGCCCCGTTGGT \\
$G b C 4 H$ & CATATCTCGCCCCGTTGGT & GCTAACCACCACGCGTTGA \\
$G b C H S$ & CCTTGACACAAGCCTTTACTCCTT & AGGGTGCGCGATCCAA \\
GbCHI & CACAACATCAG CTGTTGGATCA & CGTTTGCAGTTTCCGGTATCA \\
$G b F 3^{\prime} H$ & GCCGGCCGCAGGAT & CAATGTTGCAATAAGCAATTGGA \\
$G b F 3 H$ & ACCTTGTTGCTTCAGGACCAA & ATCCAACTCTTGCCACCATCA \\
$G b D F R$ & CGAAAGCTGAAGGGAGATACATTT & CATTGATCATTTTTGCCAGTTGA \\
$G b A N S$ & TGGTCAATGGATCACTGCAAA & CATTACTTAGAATCTCAATAGTGTC \\
& & ACCAA \\
$G b U F G T$ & AATTGAAGGCGGGAGTTTCA & AGACGATTCGTTGGATAAAACTTGT \\
$G b M Y B 1$ & GGGTGGTTATTCGGTGGTTCT & CATATAAACTTTCACCGTCTTTTTGG \\
$G b M Y B 2$ & GTTGACCAGGAGAAGGGAATTG & TGGTCATCTAAACTCTCCTCCTTTG \\
$T u b l i n$ & TGGAGGAGACCTGGCTAAGGT-3' & CGGGAGAAGACTTCAGCAACA \\
\hline
\end{tabular}
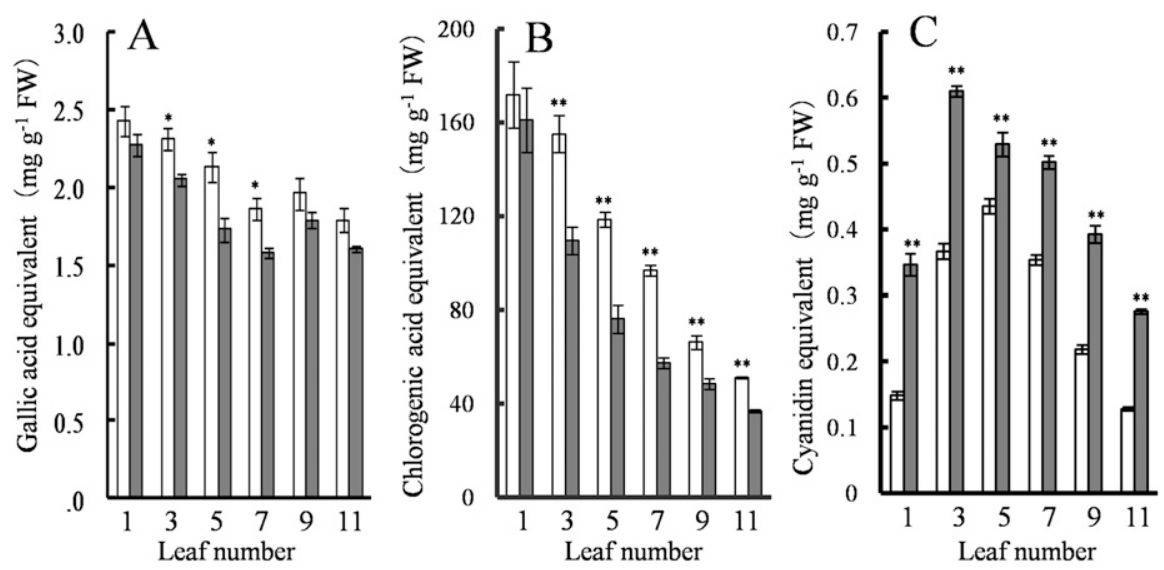

Fig. 2. Differences in phenolic content of $G$. bicolor leaves between treatments. Unshaded and solid columns indicate control and shade treatments, respectively. (A) Total polyphenol; (B) chlorogenic acid; (C) anthocyanin. Vertical bars indicate \pm SE $(\mathrm{n}=3$ or $\mathrm{n}=4)$. * and ** indicate significant differences at $P<0.05$ and $P \leq 0.01$, respectively, by Student's $t$ test. 

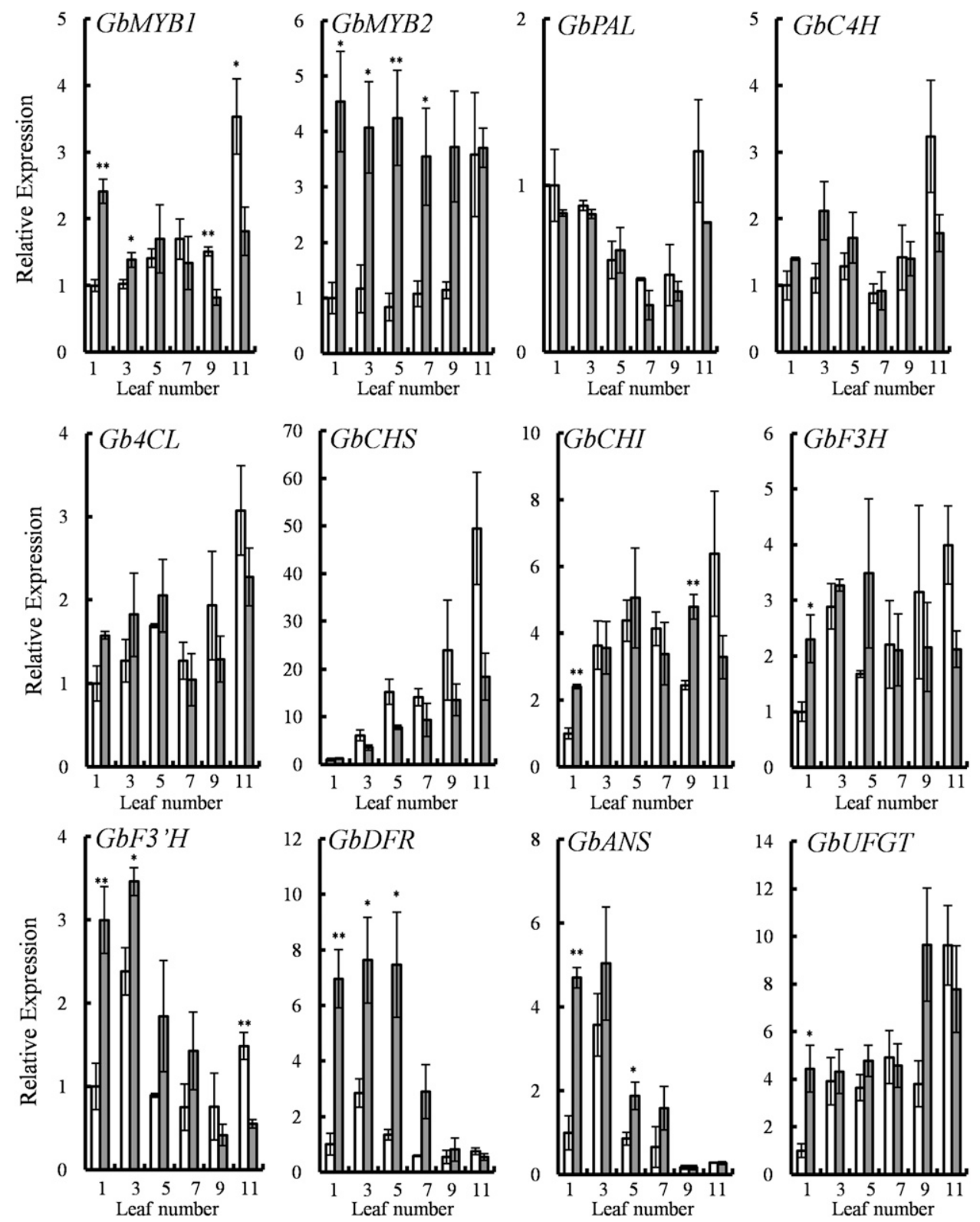

Fig. 3. Differences in gene expression of G. bicolor leaves between treatments. Unshaded and solid bars indicate control and shade treatments, respectively. Vertical bars indicate $\pm \mathrm{SE}(\mathrm{n}=4)$. ${ }^{*}$ and $* *$ indicate significant differences at $P<0.05$ and $P \leq 0.01$, respectively, by Student's $t$ test.

Antioxidant activities. Differences in antioxidant activities between the shade and control treatments are shown in Figure 4. Both the ferric-reducing power and hydroxyl radical scavenging activity became lower as the leaves matured, and lower reducing activities were detected in the shaded leaves than the control leaves regardless of leaf age.

\section{Discussion}

We found that the content of total phenolics and CGA decreased on a fresh weight basis with maturation of both control and shaded leaves. Although the anthocyanin content increased in the early stage of leaf expansion, it also decreased rapidly as leaves aged. G. bicolor leaves contain several phenolic compounds such as CGA, quercetin, kaempferol, quercitrin, isoquercitrin, and anthocyanin (Fukuoka et al.,
2012; Yoshitama et al., 1994). In the leaves of Salicaceous species (Ikonen, 2002) and Pyrus communis (Andreott et al., 2006), the phenolic composition is influenced by ontogenetic age and the content decreases with leaf age. Likewise, in leaves of Chrysobalanus icaco, anthocyanins are synthesized actively during the young expanding stage and decline significantly with leaf maturation (Nissim-Levi et al., 2003). These results suggest that leaf phenolic compounds including anthocyanins are synthesized actively in the young expanding stage and thereafter decrease with age. The loss of red color in leaves of $C$. icaco during leaf maturation is reportedly the result of dilution of anthocyanins in the expanding leaves, attributable either to masking of the red pigment by an increase in the concentration of chlorophylls or to anthocyanin degradation (Nissim-Levi et al., 2003).
The anthocyanin content in leaves is tightly dependent on environmental conditions such as light quality, light intensity, and growth temperature (Oren-Shamir, 2009). In most fruit and plant organs, light is a prerequisite for anthocyanin synthesis (Mancinelli, 1983), and the rate of anthocyanin synthesis increases with increasing light energy (Proctor, 1974). In leaves of sweetpotato, shading (i.e., $40 \%$ and $80 \%$ shading of full solar radiation) decreases anthocyanin content compared with no shading (Islam et al., 2005). However, in our study, higher anthocyanin content was detected in young expanding leaves from the shaded plot than in those from comparable ages in the control. On the other hand, temperature is also an important factor that affects anthocyanin synthesis in various fruits such as apples (Ubi et al., 2006) and grapes (Yamane et al., 2006). Many studies on the action of 

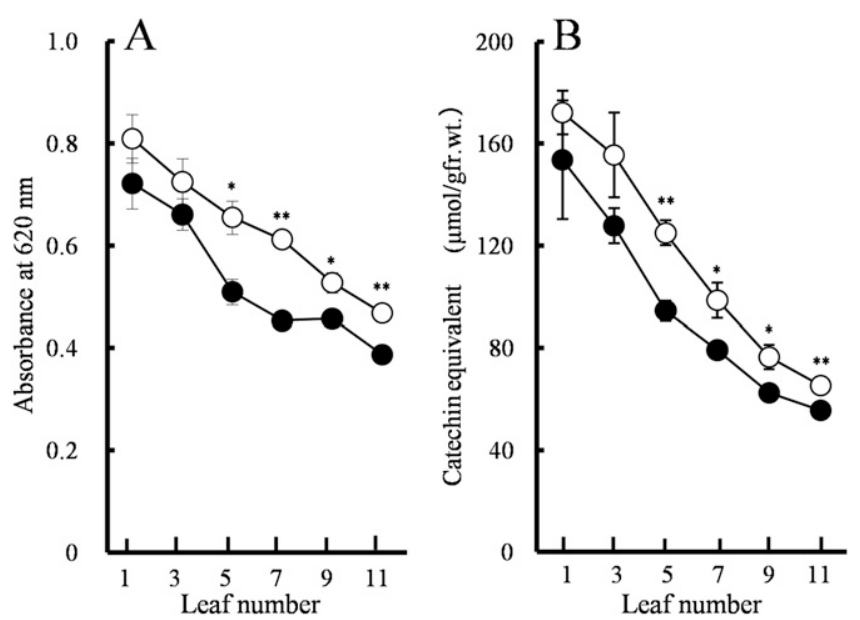

Fig. 4. Differences in antioxidant activities of $G$. bicolor leaves between treatments. Unshaded and solid circles indicate control and shade treatments, respectively. (A) Ferric ion $\left[\mathrm{Fe}\left(3^{+}\right)\right]$reducing power; (B) hydroxyl radical scavenging activity. Vertical bars indicate $\pm \operatorname{SE}(\mathrm{n}=4) .{ }^{*}$ and $* *$ indicate significant differences at $P<0.05$ and $P \leq 0.01$, respectively, by Student's $t$ test.

genes involved in anthocyanin biosynthesis in higher plants have shown that high temperature inhibits the induction of anthocyanin synthesis (Dela et al., 2003; Shvarts et al., 1997). In the Darkridge grape berry skin, continuous high night temperature inhibits anthocyanin accumulation, and this inhibition is the result of the suppression of anthocyanin biosynthetic genes including the $M Y B$-related genes (Mori et al., 2005). The expression of anthocyanin biosynthetic genes such as $C H S$ and DFR in rose (Dela et al., 2003) and CHS in apple (Lin-Wang et al., 2011) is suppressed by elevated temperatures. In our study, leaf temperatures in the control leaves reached $\approx 46^{\circ} \mathrm{C}$ in the daytime. Conversely, the temperatures in the shaded leaves were $\approx 6{ }^{\circ} \mathrm{C}$ lower than control leaves. Expression of transcription factor $G b M Y B 2$ stayed higher in shaded leaves than control leaves regardless of leaf age. The gene expressions such as $G b F 3^{\prime} H, G b D F R$, and $G b A N S$ in young expanding shaded leaves were much higher than in the control leaves of comparable age. According to Shimizu et al. (2010), anthocyanin accumulation in G. bicolor leaves is accreted in cultured sucrose-treated plantlet and this promotion is the result of increased expression of $G b D F R$ and GbANS induced by coexpression of $G b M Y B 2$ and bHLH-type transcription factor, GbMYC1. These results may suggest that red color loss in $G$. bicolor leaves in midsummer is largely dependent on the repression of GbMYB2related genes induced by high temperature. We think that shading promotes gene expressions of GbDFR and GbANS because the expression of $G b M Y B 2$ is kept high as a result of lowered temperature, and then a larger amount of anthocyanin pigments accumulates.

Generally, in higher plants, high temperatures enhance the production of ROS (Asada and Takahashi, 1987). ROS are strong oxidants that initiate local oxidative damage, leading to the disturbance of metabolic function and to the loss of cellular integrity at sites where they accumulate (Foyer et al., 1991). Tolerance to high temperatures in crop plants is associated with an increase in the activity of antioxidant enzymes (Gupta et al., 1993). Naturally occurring compounds of non-flavonoid phenolics scavenge ROS (Kanatt et al., 2005; Nara et al., 2006), and anthocyanins can also interrupt the propagation of free radicals (Galvano et al., 2004). According to Fukuoka et al. (2012), in G. bicolor leaves, a greater part of the ROS scavenging activity resulting from phenolic compounds was detected in the CGA fraction, not the anthocyanin fraction. The content of CGA is known to increase in response to environmental stresses (Oh et al., 2009). In lettuce, leaves of sun-exposed plants contained more CGA than leaves from plants growing under shaded locations (Grace et al., 1998). In this study, a larger amount of CGA was detected in young expanding control leaves than shaded leaves, and the profile of ROS scavenging activity exhibited an identical pattern to that of the CGA content. These observations suggest that increased CGA production occurs in the control leaves and that this metabolic change is an adaptive metabolic response to avoid the toxicity of ROS resulting from exposure to high temperature. We think that lowered leaf temperatures induced by shading prevent abundant ROS generation and so shaded leaves do not need to activate the CGA production to scavenge ROS.

According to Shaked-Sachray et al. (2002), both a decrease in synthesis and an increase in degradation of anthocyanin occur after a high temperature treatment. In Brunfelsia calycina flowers, the most likely candidates for the degradation of anthocyanin are enzymes such as polyphenyloxidase (PPO) and peroxidase (POD) (Vaknin et al., 2005). Induction of POD activity, resulting from high temperature, was found in the leaves of bentgrass (Liu and Huang, 2000). In Raphanus sativus, a pronounced increase in PPO activity has been observed in roots responding to elevated temperature (Fukuoka and Enomoto, 2001). Furthermore, in G. bicolor, the induction of both PPO and POD by heat stress has been reported by Fukuoka and Enomoto (2001). These results suggest that an increase in enzyme activities resulting from high temperature may play a crucial role in the enhancement of anthocyanin degradation in G. bicolor leaves. We also think that shading is an effective method to prevent temperature-induced anthocyanin degradation.

In conclusion, if $G$. bicolor plants are grown in midsummer, high temperature restricts anthocyanin synthesis but promotes CGA production in leaves. Lower production of anthocyanin pigment is the result of the repression of $G b M Y B 2$-related genes, and higher CGA production is attributed to a defense response protecting against increasing injury from ROS induced by high temperature. The artificial shading is an effective method to promote anthocyanin accumulation because the expressions of GbMYB2related genes are kept high as a result of lower temperature.

\section{Literature Cited}

Andreott, C., G. Costa, and T. Treutter. 2006. Composition of phenolic compounds in pear leaves as affected by genetics, ontogenesis and the environment. Sci. Hort. 109:130-137.

Asada, K. and M. Takahashi. 1987. Production and scavenging of active oxygen in photosynthesis, p. 227-228. In: Kyle, D.J., C.B. Osmond, and C.J. Arntzen (eds.). Photoinhibition. Elsevier, Amsterdam, The Netherlands.

Dela, G., E. Or, R. Ovadia, A. Nissim-Levi, D. Weiss, and M. Oren-Shamir. 2003. Changes in anthocyanin concentration and composition in 'Jaguar' rose flowers due to transient high-temperature conditions. Plant Sci. 164:333-340.

Foyer, C.H., M. Lelandais, E.A. Edwards, and P.M. Mullineaux. 1991. The role of ascorbate in plants, interactions with photosynthesis, and regulatory significance. Curr. Topics Plant Physiol. 6:131-144.

Fukuoka, N. and T. Enomoto. 2001. The occurrence of internal browning induced by high temperature treatment and its physiological function in Raphanus root. Plant Sci. 16:117-124.

Fukuoka, N., T. Suzuki, and Y. Yamada. 2012. Changes in polyphenol biosynthesis induced in Gynura bicolor DC. leaves by infrared irradiation. J. Hort. Sci. Biotechnol. 87:130-136.

Galvano, F., L. La Fauci, G. Lazzarino, V. Fogliano, A. Ritieni, S. Ciappellano, N.C. Battistini, B. Tavazzi, and G. Galvano. 2004. Cyanidins: Metabolism and biological properties. J. Nutr. Biochem. 15:2-11.

Grace, S.C., B.A. Logan, and W.W. Adams. 1998 Seasonal differences in foliar content of chlorogenic acid, a phenylpropanoid antioxidant, in Mahonia repens. Plant Cell Environ. 21:513-521.

Gupta, A.S., R.P. Webb, A.S. Holaday, and R.D. Allen. 1993. Overexpression of superoxide dismutase protects plants from oxidative stress. Induction of ascorbate peroxidase in superoxide dismutase overexpressing plants. Plant Physiol. 103:1067-1073.

Hayashi, M., S. Miwa, T. Yagi, T. Michihata, and T. Enomoto. 2002. Antioxidative activity of extract from Kinjisou (Gynura bicolor DC) and its effective use technology for various foods. Bull. Ishikawa Agr. Res. Center. 24:25-33.

Ikonen, A. 2002. Preferences of six leaf beetle species among qualitatively different leaf age class off three Salicaceous hot species. Chemoecol. 12:23-28. 
Islam, M.S., M. Jalaluddin, and J.O. Garner. 2005. Artifical shading and temperature influence on anthocyanin compositions in sweetpotato leaves. HortScience 40:176-180.

Kanatt, S.R., R. Chander, P. Radhakrishina, and A. Sharma. 2005. Potato peel extract-A natural antioxidant for retarding lipid peroxidation in radiation processed lamb meat. J. Agr. Food Chem. 53:1499-1504.

Koyama, K. and N. Goto-Yamamoto. 2008. Bunch shading during different developmental stages affects the phenolic biosynthesis in berry skins of 'Cabernet Souvignon' grapes. J. Amer. Soc. Hort. Sci. 133:743-753.

Lin-Wang, K., D. Micheletti, J. Palmer, R. Volz, R. Lozano, R. Espley, R. Hellens, D. Chagne, D. D. Rowan, M. Troggio, I. Iglesias, and A.C. Allan. 2011. High temperature reduces apple fruit colour via modulation of the anthocyanin regulatory complex. Plant Cell Environ. 34:11761190.

Liu, X. and B. Huang. 2000. Heat stress injury in relation to membrane lipid peroxidation in creeping bentgrass. Crop Sci. 40:503-510.

Mancinelli, A.L. 1983. The photoregulation of anthocyanin synthesis, p. 640-661. In: Shropshire, W., Jr. and H.H. Mohr (eds.). Photomorphogenesis. Springer-Verlag, Berlin, Germany.

Mori, K., N. Goto-Yamamoto, M. Kitayama, and K. Hashizume. 2007. Loss of anthocyanins in red-wine grape under high temperature. J. Expt. Bot. 58:1935-1945.

Mori, K., S. Sugaya, and H. Gemma. 2005. Decreased anthocyanin biosynthesis in grape berries grown under elevated night temperature condition. Sci. Hort. 105:319-330.

Nara, K., T. Miyoshi, T. Honma, and H. Koga. 2006. Antioxidative activity of bound-form phenolics in potato peel. Biosci. Biotechnol. Biochem. 70:1489-1491.

Nissim-Levi, A., S. Kagan, R. Ovadia, and M. OrenShamir. 2003. Effects of temperature, UV-light and magnesium on anthocyanin pigmentation in cocoplum leaves. J. Hort. Sci. Biotechnol. 78:61-64.

Oh, M.M., E.E. Carey, and C.B. Rajashekar. 2009. Environmental stress induce health-promoting phytochemicals in lettuce. Plant Physiol. Biochem. 47:578-583.

Oren-Shamir, M. 2009. Does anthocyanin degradation play a significant role in determining pigment concentration in plants? Plant Sci. 177:310-316.

Proctor, J.T.A. 1974. Color stimulation in attached apples with supplementary light. Can. J. Plant Sci. 54:499-503.

Shaked-Sachray, L., D. Weiss, M. Reuveni, A. NissimLevi, and M. Oren-Shamir. 2002. Increased anthocyanin accumulation in aster flowers at elevated temperatures due to magnesium treatment. Plant Physiol. 114:559-565.

Shimizu, Y., K. Maeda, M. Kato, and K. Shimomura 2010. Isolation of anthocyanin-related MYB gene, GbMIB2, from Gynura bicolor leaves. Plant Biotechnol. 27:481-487.

Shvarts, M., A. Borochov, and D. Weiss. 1997. Low temperature enhances petunia flower pigmentation and induces chalcone synthase gene expression. Physiol. Plant. 99:67-72.
Singleton, V.L., R. Oethofer, and R.M. LamuelaRaventos. 1999. Analysis of total phenols and other oxidation substrates and antioxidants by means of Folin-Ciocalteau reagent. Methods Enzymol. 299:152-278.

Ubi, B.E., C. Honda, H. Bessho, S. Kondo, M. Wada S. Kobayashi, and T. Moriguthi. 2006. Expression analysis of anthocyanin biosynthetic genes in apple skin: Effect of UV-B and temperature. Plant Sci. 170:571-578.

Vaknin, H., A. Bar-Akiva, R. Ovadia, A. NissimLevi, I. Forer, D. Weiss, and M. Oren-Shamir. 2005. Active anthocyanin degradation in Brunfelsia calycina (yesterday-today-tomorrow) flowers. Planta 222:19-26.

Wang, H., O. Arakawa, and Y. Motomura. 2000. Influence of maturity and bagging on the relationship between anthocyanin accumulation and phenylalanine ammonia-lyase (PAL) activity in 'Jonathan' apples. Postharvest Biol. 19:123-128.

Yamane, T., S.T. Jeong, N. Goto-Yamamoto, Y. Koshita, S. Kobayashi, and S. Obayashi. 2006. Effects of temperature on anthocyanin biosynthesis in grape berry skins. Amer. J. Enol. Viticult. 57:54-59.

Yoshiaki, A., M. Murahama, Y. Okada, and Y. Yamada. 2010. Effect of thermal condition on plant growth of Kinjisou (Gynura bicolor DC). Abst. Japan Soc. Hort. Sci. Hokuriku Meet. p. 35.

Yoshitama, K., M. Kaneshige, N. Ishikura, F. Araki, S. Yahara, and K. Abe. 1994. A stable reddish purple anthocyanin in the leaf of Gynura aurantiaca cv. 'Purple Passion'. J. Plant Res. 107:209-214. 